\title{
Pseudo-Hermiticity versus PT-symmetry. II. A complete characterization of non- Hermitian Hamiltonians with a real spectrum
}

Ali Mostafazadeh

Citation: Journal of Mathematical Physics 43, 2814 (2002); doi: 10.1063/1.1461427

View online: http://dx.doi.org/10.1063/1.1461427

View Table of Contents: http://aip.scitation.org/toc/jmp/43/5

Published by the American Institute of Physics

\section{Articles you may be interested in}

Pseudo-Hermiticity versus PT symmetry: The necessary condition for the reality of the spectrum of a non-

Hermitian Hamiltonian

Journal of Mathematical Physics 43, 205 (2001); 10.1063/1.1418246

Pseudo-Hermiticity versus PT-symmetry III: Equivalence of pseudo-Hermiticity and the presence of antilinear symmetries

Journal of Mathematical Physics 43, 3944 (2002); 10.1063/1.1489072

Pseudo-Hermiticity and generalized PT- and CPT-symmetries

Journal of Mathematical Physics 44, 974 (2003); 10.1063/1.1539304

PT-symmetric quantum mechanics

Journal of Mathematical Physics 40, 2201 (1999); 10.1063/1.532860

Must a Hamiltonian be Hermitian?

American Journal of Physics 71, 1095 (2003); 10.1119/1.1574043

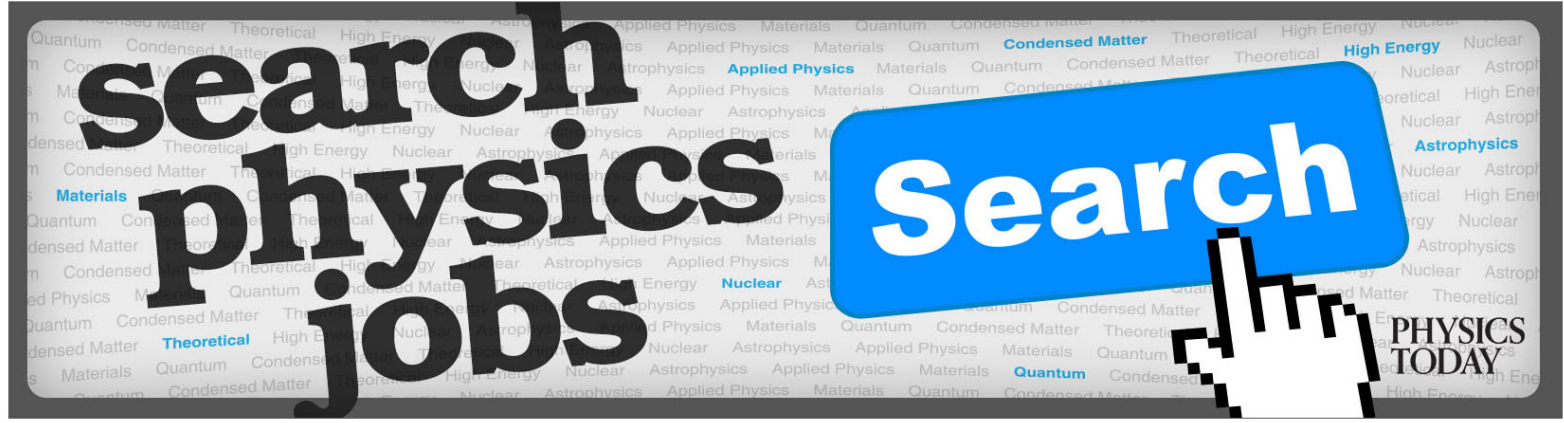




\title{
Pseudo-Hermiticity versus $\boldsymbol{P T}$-symmetry. II. A complete characterization of non-Hermitian Hamiltonians with a real spectrum
}

\author{
Ali Mostafazadeh ${ }^{\text {a) }}$ \\ Department of Mathematics, Koç University, Rumelifeneri Yolu, \\ 80910 Sariyer, Istanbul, Turkey
}

(Received 17 October 2001; accepted for publication 28 January 2002)

We give a necessary and sufficient condition for the reality of the spectrum of a non-Hermitian Hamiltonian admitting a complete set of biorthonormal eigenvectors. (C) 2002 American Institute of Physics. [DOI: 10.1063/1.1461427]

Recently, we have explored in Ref. 1 the basic mathematical structure underlying the spectral properties of $P T$-symmetric Hamiltonians. ${ }^{2}$ In particular, we have shown that these properties are associated with a class of more general (not necessarily Hermitian) Hamiltonians $H$ satisfying

$$
H^{\dagger}=\eta H \eta^{-1}
$$

where a dagger denotes the adjoint of the corresponding operator and $\eta$ is a Hermitian invertible linear operator. We have termed such a Hamiltonian " $\eta$-pseudo-Hermitian." Hermitian and the $P T$-symmetric Hamiltonians that admit a complete set of biorthonormal eigenvectors constitute subsets of the set of pseudo-Hermitian Hamiltonians. For a $P T$-symmetric Hamiltonian, the exactness of $P T$-symmetry ensures the reality of the energy spectrum. The purpose of this article is to provide a complete characterization of the Hamiltonians that have a real spectrum assuming that they are endowed with a complete set of biorthonormal eigenvectors.

By definition, a $P T$-symmetric Hamiltonian has a symmetry given by an antilinear operator, namely $P T$. It is well-known that if a Hamiltonian satisfies

$$
[H, A]=0,
$$

for an anti-linear operator $A$, then

$(\star)$ either the eigenvalues of $H$ are real or they come in complex conjugate pairs.

Furthermore, an eigenvalue of $H$ is real provided that a corresponding eigenvector is invariant under the action of $A$, i.e., Eq. (2), together with

$$
H|E\rangle=E|E\rangle,
$$

and

$$
A|E\rangle=|E\rangle
$$

imply $E \in \mathbb{R}$. Therefore, a Hamiltonian with an antilinear symmetry has a real spectrum if the symmetry is exact.

In Ref. 1 , we have shown that every pseudo-Hermitian Hamiltonian has the property $(\star)$.

Furthermore, for Hamiltonians with a complete set of biorthonormal eigenvectors this property is the necessary and sufficient condition for pseudo-Hermiticity. This, in particular, means that pseudo-Hermiticity is a necessary condition for having a real spectrum, but it is not sufficient. In the following we give the necessary and sufficient condition for the reality of the spectrum of any

${ }^{a)}$ Electronic mail address: amostafazadeh@ku.edu.tr 
Hamiltonian that admits a complete set of biorthonormal eigenvectors. We shall only consider the case of discrete spectra. The generalization to continuous spectra does not seem to involve major difficulties.

We first recall the defining properties of a Hamiltonian admitting a complete set of biorthonormal eigenvectors. ${ }^{3}$ If a Hamiltonian $H$ has a complete set of biorthonormal eigenvectors $\left\{\left|\psi_{n}\right\rangle,\left|\phi_{n}\right\rangle\right\}$, then

$$
\begin{gathered}
H\left|\psi_{n}\right\rangle=E_{n}\left|\psi_{n}\right\rangle, \quad H^{\dagger}\left|\phi_{n}\right\rangle=E_{n}^{*}\left|\phi_{n}\right\rangle, \\
\left\langle\phi_{m} \mid \psi_{n}\right\rangle=\delta_{m n}, \\
\sum_{n}\left|\psi_{n}\right\rangle\left\langle\phi_{n}\right|=1,
\end{gathered}
$$

where $n$ is the spectral label, $\delta_{m n}$ denotes the Kronecker delta function, and 1 is the identity operator.

Theorem: Let $H: \mathcal{H} \rightarrow \mathcal{H}$ be a Hamiltonian that acts in a Hilbert space $\mathcal{H}$, has a discrete spectrum, and admits a complete set of biorthonormal eigenvectors $\left\{\left|\psi_{n}\right\rangle,\left|\phi_{n}\right\rangle\right\}$. Then the spectrum of $H$ is real if and only if there is an invertible linear operator $O: \mathcal{H} \rightarrow \mathcal{H}$ such that $H$ is $O O^{\dagger}$-pseudo-Hermitian.

Proof: Let $\{|n\rangle\}$ be a complete orthonormal basis of $\mathcal{H}$, i.e.,

$$
\langle m \mid n\rangle=\delta_{m n}, \quad \sum_{n}|n\rangle\langle n|=1,
$$

and $O: \mathcal{H} \rightarrow \mathcal{H}$ and $H_{0}: \mathcal{H} \rightarrow \mathcal{H}$ be defined by

$$
O:=\sum_{n}\left|\psi_{n}\right\rangle\left\langle n\left|, \quad H_{0}:=\sum_{n} E_{n}\right| n\right\rangle\langle n| .
$$

Then, in view of (5)-(9), $O$ is invertible with the inverse given by

$$
O^{-1}=\sum_{n}|n\rangle\left\langle\phi_{n}\right|
$$

and

$$
O^{-1} H O=H_{0} .
$$

Now suppose that the spectrum of $H$ is real. Then, $H_{0}$ is Hermitian, and taking the adjoint of both sides (11), we have

$$
O^{-1} H O=O^{\dagger} H^{\dagger} O^{-1 \dagger}
$$

or alternatively

$$
H=\left(O O^{\dagger}\right) H^{\dagger}\left(O O^{\dagger}\right)^{-1} .
$$

This equation shows that $H$ is $O O^{\dagger}$-pseudo-Hermitian. This completes the proof of necessity. Next we suppose that $H$ is $O O^{\dagger}$-pseudo-Hermitian. Then (13) and consequently (12) hold. On the other hand, in view of (6) and (9), we have

$$
H_{0}=O^{-1} H O, \quad H_{0}^{\dagger}=O^{\dagger} H^{\dagger} O^{-1 \dagger} .
$$

Therefore, (12) implies that $H_{0}$ is Hermitian, and the eigenvalues $E_{n}$ are all real. 
It should be emphasized that the characterization of the non-Hermitian Hamiltonians with a real spectrum given by the preceding theorem applies to the Hamiltonians that admit a complete biorthonormal system of eigenvectors. A generalization of this result to the case of arbitrary Hamiltonians is not known.

The basic idea of the present work was originated from a comment made by Z. Ahmed about his checking the results the author had reported in Ref. 1 for a specific example of a non-Hermitian Hamiltonian with a real spectrum. This project was supported by the Young Researcher Award Program (GEBIP) of the Turkish Academy of Sciences.

${ }^{1}$ A. Mostafazadeh, J. Math. Phys. 43, 205 (2002).

${ }^{2}$ C. M. Bender and S. Boettcher, Phys. Rev. Lett. 80, 5243 (1998); F. M. Fernández, R. Guardiola, J. Ros, and M. Znojil, J. Phys. A 31, 10105 (1998); F. Cannata, G. Junker, and J. Trost, Phys. Lett. A 246, 219 (1998); C. M. Bender, S. Boettcher, and P. N. Meisenger, J. Math. Phys. 40, 2201 (1999); C. M. Bender, G. V. Dunne, and P. N. Meisenger, Phys. Lett. A 252, 272 (1999); C. M. Bender and G. V. Dunne, J. Math. Phys. 40, 4616 (1999); G. A. Mezincescu, J. Phys. A 33, 4911 (2000); E. Delabaere and D. T. Trinh, ibid. 33, 8771 (2000); B. Bagchi and R. Roychoudhury, ibid. 33, L1 (2000); A. Khare and B. P. Mandal, Phys. Lett. A 272, 53 (2000); B. Bagchi, F. Cannata, and C. Quesne, ibid. 269, 79 (2000); M. Znojil, F. Cannata, B. Bagchi, and R. Roychoudhury, Phys. Lett. B 483, 284 (2000); M. Znojil and M. Tater, J. Phys. A 34, 1793 (2001); C. M. Bender, G. V. Dunne, P. N. Meisenger, and M. Şimşek, Phys. Lett. A 281, 311 (2001); F. Cannata, M. Ioffe, R. Roychoudhury, and P. Roy, ibid. 281, 305 (2001); Z. Ahmed, ibid. 282, 343 (2001); 284, 231 (2001); 290, 19 (2001); P. Dorey, C. Dunning, and R. Tateo, LANL Archives: hep-th/0104119; M. Znojil, LANL Archives: hep-th/0101038; M. Znojil, LANL Archives: math-ph/0104012; G. S. Japaridze, LANL Archives: quant-ph/0104077; R. Kretschmer and L. Szymanowski, LANL Archives: quant-ph/0105054.

${ }^{3}$ J. Wong, J. Math. Phys. 8, 2039 (1967); F. H. M. Faisal and J. V. Moloney, J. Phys. B 14, 3603 (1981). 